(C) 2006 IEEE. Personal use of this material is permitted. Permission from IEEE must be obtained for all other uses, in any current or future media, including reprinting/republishing this material for advertising or promotional purposes, creating new collective works, for resale or redistribution to servers or lists, or reuse of any copyrighted component of this work in other works. 


\title{
Creating Affective Visualisations for a Physiologically Interactive Artwork
}

\author{
Lizzie Muller*, Greg Turner*, George Khut† \& Ernest Edmonds* \\ *Creativity and Cognition Studios, University of Technology Sydney, \\ $\dagger$ University of Western Sydney
}

lizzie@lizziemuller.com,greg@gregturner.org,george@georgekhut.com, ernest@ernestedmonds.com

\begin{abstract}
This paper describes an action research project based on the process of designing the visualisation of heart and breath rate data for the interactive artwork Cardiomorphologies. The project aimed to realise the affective goals of the artist as closely as possible by studying the audience experience of the visualisations and incorporating the findings into an iterative design process. The qualities of richness and ambiguity were found to contribute to the achievement of the artists' two major aims: 1) to create a sense of integrated physical and mental engagement with the work and 2) to create a reflective state in which participants consider correlations between their thoughts and specific physiological states 1 .
\end{abstract}

\section{Introduction}

Creating unusual, evocative and thought provoking ways of representing different kinds of information is central to a great deal of computer-based contemporary art practice $[1,2]$. In artistic contexts the affective qualities of these visualisations are at least as important as their ability to convey information efficiently and accurately. The simulation and provocation of affective states is becoming increasingly desirable in HumanComputer Interaction studies in general as the number of personal communication and entertainment technologies increases [3, 4]. How people feel whilst using these technologies is of growing importance. Several researchers have pointed out that studying interactive art has a key role to play in understanding and designing affective human-computer interaction $[5,6]$.

To design and evaluate affective qualities of an artistic visualisation it is necessary to consider first and foremost the participating audience's experience. Understanding audience experience becomes even more critical in the case of physiologically responsive artworks such as
Cardiomorphologies by George Khut, which creates audiovisual representations of the participant's breath and heart rate. The information represented in this work is drawn from the participant themselves and a complex feedback loop is created in which the data visualisation both provokes and reflects the participant's reaction to it.

This paper reports on an action research project which studied the audience's experience of Cardiomorphologies and incorporated it into the process of designing visualisations of the breath and heart. The aim was to realise as far as possible the experiential and affective goals desired by the artist. The paper demonstrates how the use of richness and ambiguity in the design contributed to the goals of engagement and reflection. It shows how these qualities relate to the way in which participants approach, experience and interpret information.

\section{Background and Aims}

Cardiomorphologies is part of a series of artworks by George Khut which use biofeedback technologies [7]. Khut aims to give audiences an alternative insight into the way our thoughts and physical sensations are intimately linked. Specifically, he aims to enable participants to influence their heart rates through experimenting with their breathing and with their thoughts. Seeing and hearing their heart and breath helps the participants to perceive, focus on and monitor physiological changes which they may not have the ability to detect or recognise from sensation alone. Khut's work is part of a tradition of artists' experimentation with physiological and biofeedback technologies which stretches back to the 1960's [5, 8]. 


\subsection{Cardiomorphologies}

Individuals participating in Cardiomorphologies are seated on a comfortable reclining chair in a dimly lit and enclosed space. The participant is fitted with a breath sensor (respiratory strain gauge) and heart beat sensor (EKG). Graphic visualisations are displayed on a large screen in front of the participant and a sonification of the same cardio-respiratory data is projected through a quadraphonic speaker array. The heart sensor produces a 'pulse' signal for every heart beat. The breath sensor produces a value which increases the more the sensor (strapped around the ribcage) is stretched. These changes are analysed to produce various readings and statistics, the most important analyses focus on the subject's heart rate variations (HRV) and the relative amplitudes of the various low frequency oscillations that constitute the moment-to-moment changes in HRV.

The participants' perception of these changes in heart rate is fundamental to the experiential aim of Cardiomorphologies, especially with regard to the influence of breath on these patterns. The physiological data to be visualised, then, is simply the state of the heart and breath over time which are easily represented and comprehended as simple numerical values or points on a chart. The challenge for the artist and research team was to infuse what could be a straightforward read-out of numbers with the affective qualities that the artist wants the audience to experience.

\subsection{Affective aims of the artist}

There are two key aspects to the audience experience that Khut is trying to create with Cardiomorphologies. Firstly a sense of integrated physical and mental engagement with the work and secondly a reflective state in which participants consider correlations between thoughts and specific physiological states In order to structure the research process and to judge success Khut, together with the research team, established a set of affective aims at the outset of the project which articulated these experiential goals as clearly as possible. To describe the sense of physical and mental engagement the team chose:

i) Sensual and kinaesthetic: the projected imagery generates and reinforces enjoyable sensations of changing weight, motion and patterning within the body.

ii) Close fitting: the artwork should reflect as accurately as possible actual changes in physiology. It should feel like a very clear extension of the participant's body.

iii) Quiet, concentrated, inwardly-attentivefocus: the participant is focused on sensing and responding to subtle shifts within their own psycho-physiology.

And to describe a state of reflection:

iv) Explorative, curious: the experience invites exploration and interest.

v) Enabling: participants develop an ability to physically sense changes in heart rate pattern, and how these patterns interact with different mental/emotional states.

vi) Instructive: participants have a sense that they have learned something about their own ability to sense and enact changes in their own psychophysiology, as revealed to them through self initiated changes in their own cardio-respiratory processes.

vii) Meaningful: the artwork provokes a consideration of the participant's mindbody processes in ways that might inform their experiences and actions in other 'real life' contexts.

\section{Research methodology}

This study is part of a series of investigations into the audience experience of interactive artworks that have taken place using Beta_space, an experimental public environment for interactive art at the Powerhouse Museum, Sydney [9-11]. A data gathering method for working in public exhibition spaces, based on video-cued recall, has been developed through several Beta_space studies, and is described briefly below. In this study of Cardiomorphologies an action research approach was adopted, where the experiential data gathered was used to drive a process of formative evaluation in which the artwork was refined over two iterations.

\subsection{Video-cued recall method}

This method of data gathering requires the participant to watch a video of him/herself interacting with an artwork and to simultaneously give an account of their experience. It aims to elicit richly descriptive verbal data rooted in real lived experience [12]. The method enables participants to recall pre-verbal perceptual, motivational and affective states that rarely emerge from interview data alone $[4,13,14]$. With Cardiomorphologies 
there were some specific challenges in the use of this method. In previous studies it was noted that participants seeing their own movements was an important factor in prompting recall [9]. In Cardiomorphologies the participant is almost completely still, which could make it difficult for participants to link exact details of experience to specific moments in the video. After the reports all participants were questioned about how easy or difficult they found the process, and how accurate they judged their accounts to be. The majority felt that the sequence of memories had usually been more or less accurate and that the visuals had prompted specific memories at specific points. However not all were sure that they correctly identified the right memory at the right moment in the tape, and there was a good deal of selfcorrection in which people reported a memory and then, seconds later, re-identified the moment when the memory occurred.

Data was collected from ten people using video-cued recall, five in the first iteration (two women and three men) and five in the second (three women and two men). Participants were of varying ages, with diverse professional and personal circumstances.

In analysing the data from this technique Khut's affective aims were used as a guideline. All comments relating to the desired states (either positive or negative) were highlighted and linked as closely as possible to the aspect of the piece which had provoked it.

\subsection{Participatory design: focus group method}

A second research process was drawn from the field of participatory design [15] to allow collaboration with the audience based on their experience of the work. A group of six participants (three men and three women of varying ages) experienced Cardiomorphologies over the course of the day, and then joined together for a structured group discussion.

The discussion began with the generation of shared descriptive language. For this phase participants took it in turns to describe the experience of the work as it unfolded over the course of their interaction. The rest of the group listened and noted down key phrases that concurred with or contradicted their own experience of the work. They marked the phrases as either positive or negative.

The artist's experiential and affective goals were introduced in the second stage. Participants were asked to assign words and phrases generated in the first stage to different goals. The aim was to find out whether any of the goals coincided with the participants' own understanding of their experience, and, if so, what language they used to describe those experiences and what aspects of them were positive or negative.

\section{The Design Process}

This section describes the process of working with audience data to refine the visualisations, including the initial visualisation strategy, the lessons learned from the audience, and the improvements implemented in response.

\subsection{Visualisation strategy}

For the first iteration three lightweight visualisations called cross mode, rings mode and spectral graph were designed. Cross mode showed the breath and heart rate on an XY axis, which expanded and contracted in real-time (see figure 1). The aim of cross mode was to provide a very clear and easy to read visualisation that participants could quickly understand.

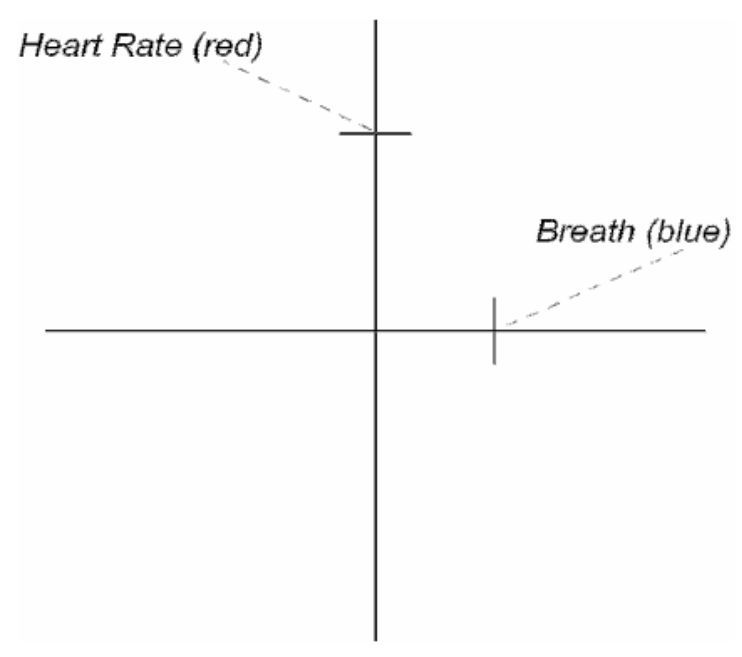

Figure 1. Monochrome redrawing of Cross mode (the descriptive text was not shown)

Rings mode provided a more abstract visualisation of the heart and breath rate in (see figure 2). A simple blue ring expanded and contracted with the breath. This ring would appear softer and larger with slower breath rates, and harder and thinner with faster breath rates. A red ring represented the heart rate - the slower the rate the smaller the ring. The aim of the rings was to try and create a relationship between physical (proprioceptive) sensation and visualisation. The expansion and 
contraction of the rings had an analogous relationship to the expansion and contraction of the ribs/abdomen with the breath, and metaphorically suggested the cycles of respiration and blood circulation.

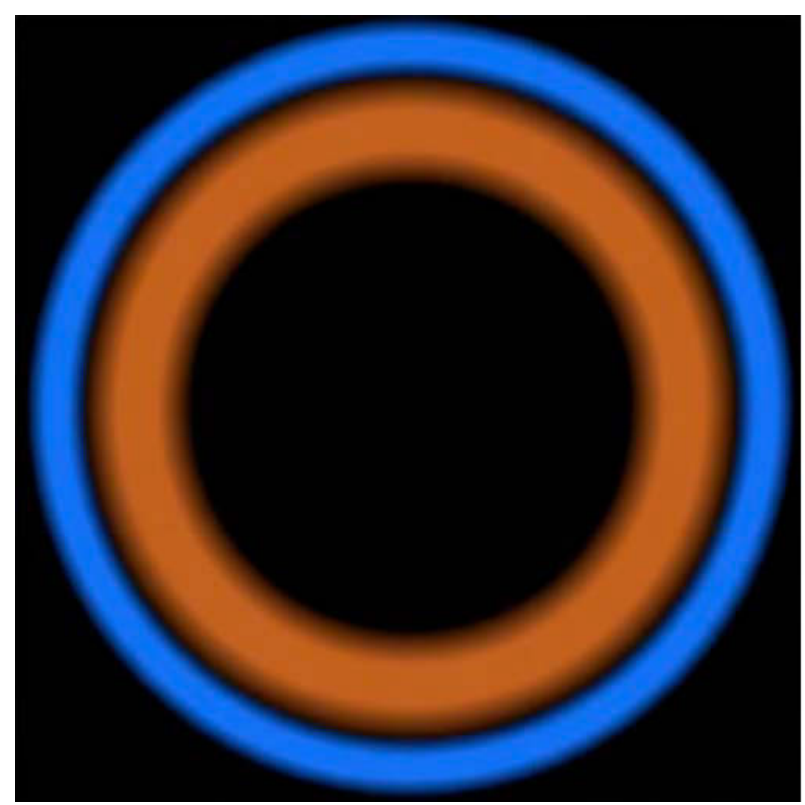

Figure 2. Rings mode

The spectral graph visualisation showed the heartrate- variability spectrum data (see figure 3), which was more complicated, and less immediate than the information presented in the simpler visualisations, but in theory can be more representative of the participant's affective state $[16,17]$. This part of the data was very important to the artist as it showed how psychological orientation, such as sustaining a thought about a relaxing or stressful situation could stimulate different physiological responses.

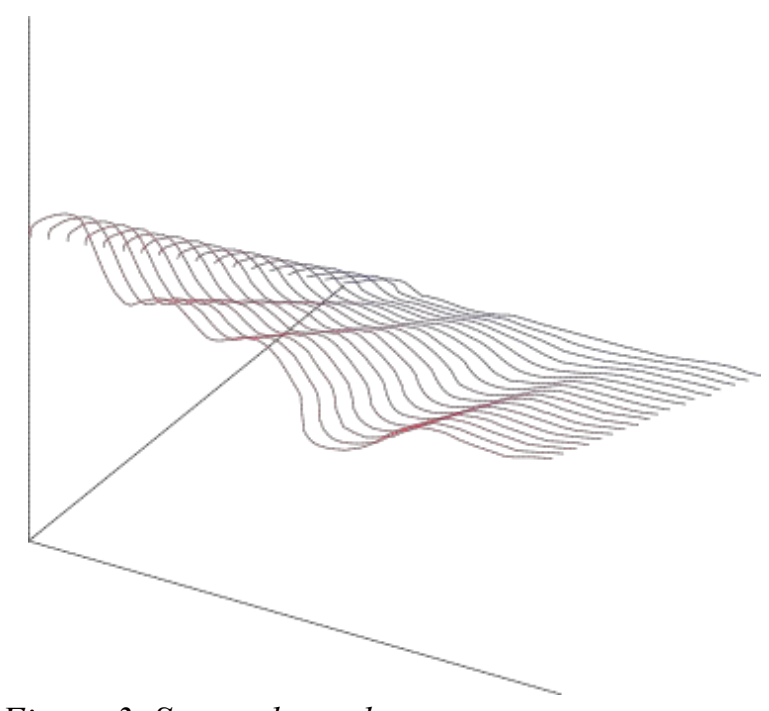

Figure 3. Spectral graph

\subsection{Data Filtering and Shaping}

During the first iteration some technical problems were immediately observed which manifested themselves with novice participants. The sensors were difficult to attach and calibrate for the wide variety of body shapes, particularly when the person attaching the sensor is physically distant from the signal calibration displays. Once attached, it was easy for audiences to produce noisy data by moving the sensors, which mostly happened at the beginning and the end of each session, but occasionally during. The heart sensor occasionally missed a beat, which not only corrupts the HRV analysis, but can be very disconcerting for a participant used to hearing and seeing the regularity of their heartbeat.

To address these problems in the second version, the process of filtering the data was refined and the transitions between phases (the attaching and removing of sensors and introducing new display elements) was smoothed.

The reliability of the data was improved firstly by moving from an electrode method for collecting heart data to a handheld device such as those used in exercise environments. In software terms the problem was approached by making an audio calibration tool and using low-pass filtering, limiting and damping to reduce noise. The erroneous HRV spectrum data at the beginning of each session was hidden, and the visualisation was introduced with a slow fade after the required five minutes needed to produce an accurate analysis of the data.

\subsection{Richness}

All the participants found the cross visualisation easy to understand (demonstrated in short affirmations like "there's my breath"), but in most cases it made very little impact on them. Most didn't comment on this visualisation in any depth (in contrast to their response to the other visualisations described below). The responses to rings mode were markedly different. The ring visualisations commanded the attention of the participants, and their reports were more descriptive and more strongly positive. There is evidence in the reports that the rings encouraged experimentation and play, which deepened engagement:

"at this point...I'm all engaged by the circles... I think they're amazing and I was trying to experiment with my own breathing to see how much of the shape I can sustain [...] 


\section{I'm trying to create something with my breathing here. It's a very joyful experience”.}

The focus group confirmed the engaging effect of the rings (which they described as doughnuts) as positively contributing to more than one of the artist's affective goals. Under the category of Quiet, Concentrated, Inward two participants described them as hypnotic, under Sensual and Kinaesthetic they were described as comfortable, and under Explorative/Curious they were described as playful and absorbing.

A key part of the appeal and the limitation of the rings was the colour, several participants commented on how visually attractive the colours were, but several also became bored with the lack of variation, and wished the colours would change. This feedback suggested that the visualisations needed to offer the participants varied and creative possibilities, whilst not undermining the hypnotic and concentrated simplicity they found in the rings. Richness needed to be added, but not complication. As a result cross mode was abandoned and ring mode was developed, including the addition of richer colour variations and effects including fading, blurring and deepening of different colours depending on heart rate and breathing patterns. The result evoked a lens flare or ball lightning effect which gave rise to a whole new set of variables from the additive blending of colours and contours which created an extensive palette from which participants could create highly individual patterns.

\subsection{Ambiguity}

While few participants expressed any confusion about the information represented by the rings, there was a great deal of confusion expressed over the spectral graph. Three members of the focus group put lack of understanding of the graph as negatively impacting on the category of Close Fit, and it was also described negatively under Quiet, Concentrated, Inwardly attentive focus.

However the overall response to this visualisation was ambivalent. There were a number of positive responses to the graph based on the attractiveness of its appearance under Sensual/Kinaesthetic. Within the video-cued recall data there was evidence that for both the participants that did understand the visualisation and those that didn't the appearance of the graph prompted experimentation with different kinds of thoughts and an intense effort to interpret their physiological responses.
This indicated that there was value in the aesthetic and suggestive qualities of this visualisation but that it was very difficult for people to interpret exactly what it represented. As a result it interfered with their sense of direct connection with the work.

At the same time the focus group suggested that several of the affective categories under Khut's second experiential goal; a reflective approach, were not being achieved. Participants linked very few of their responses to the categories Enabling, Instructive and Meaningful, and reported that these aims did not match their experience of the work. Interestingly they also reported that they did not see these aspects as necessarily desirable.

It seemed that the expectations and designs of the artist and the research group were perhaps too literal or didactic for the artistic context. Audiences couldn't interpret their physiological data very accurately, and were also not driven by a need to understand precisely what the data meant. The solution was to experiment with the use of ambiguity as a positive design virtue to encourage both engagement and reflection. Gaver et al [18] have argued that ambiguity can be used as a resource for design, especially in affective realms such as art. They argue that the uncertain meanings in ambiguous systems lead people to interpret situations for themselves and consequently develop deeper and more personal relations with the meanings offered by those systems. Further than this they argue that ambiguous systems are "evocative rather than didactic and mysterious rather than obvious".

Their arguments suggest that ambiguity could be used positively to achieve both engagement, and the seemingly elusive experiential aim of a reflective state.

As a result the graph was transformed into the more abstract representation by having the spectra appear like rays from the centre of the rings after five minutes of the experience (see figure 4). This delay allowed for the collection of enough meaningful data from the participant to create a true spectral display, but also was a means to stage the introduction of new kinds of suggestive information. The rays thus became an invitation rather than an instruction to consider psychophysiological changes over time without breaking the direct connection with the heart and breath rate that is so important to the sense of Close Fit which creates engagement. 


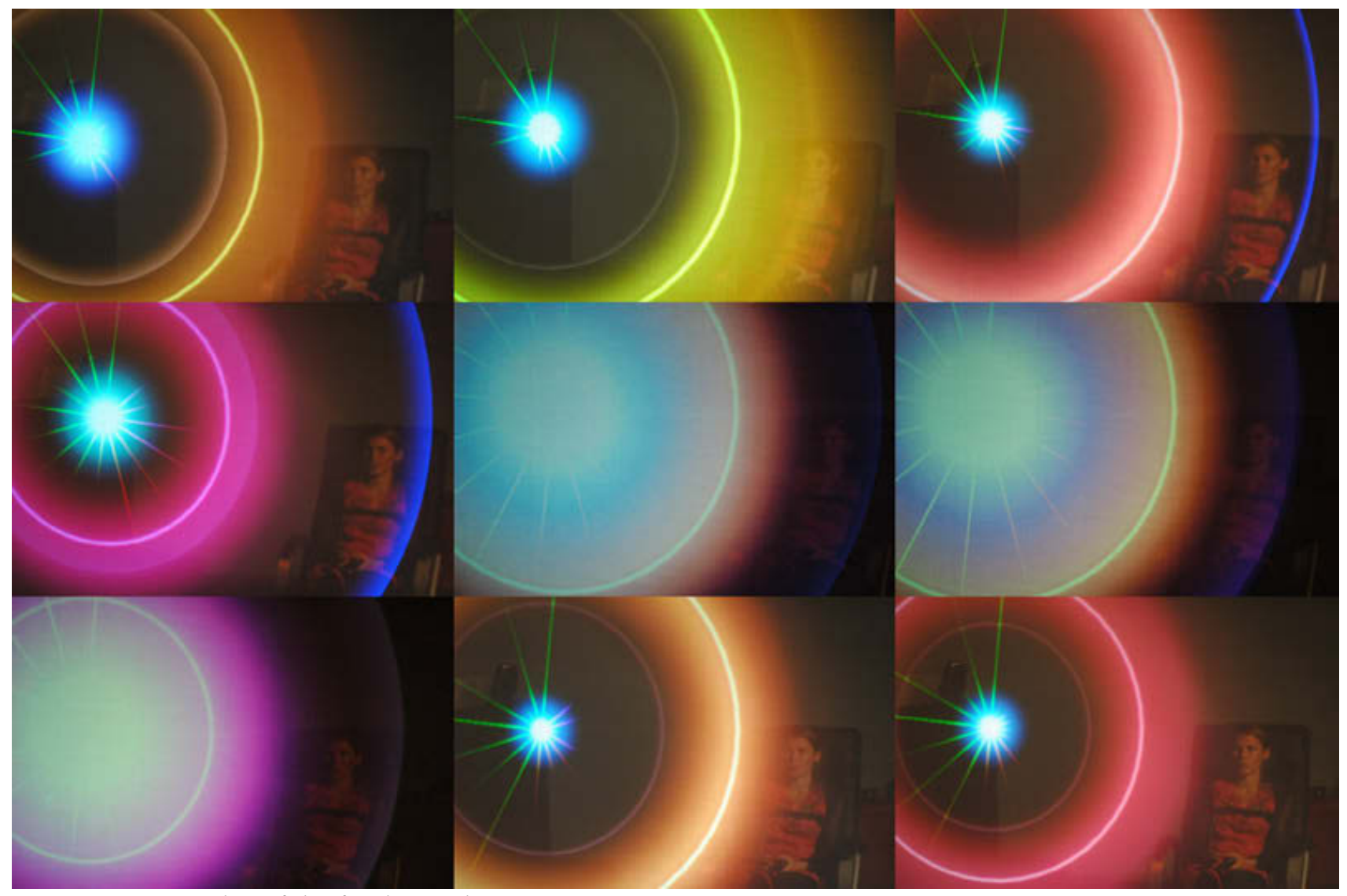

Figure 4: Examples of the final visualization

\section{Results}

The experiences reported by the participants in the final version of Cardiomorphologies included intensely personal feelings and emotions, which demonstrated the affective power of the visualisations. Their accounts revealed the extent to which Khut's experiential aims of engagement and reflection had been achieved, and also some important insights into the role of richness and ambiguity in this success.

\subsection{Engagement}

There was a great deal of evidence to suggest that the aims of Quiet, Concentrated and Inwardly Attentive Focus, and Sensual and Kinaesthetic were achieved. After a period expectation at the start, all reported feeling relaxed and calm. The majority of participants reported that they were focused on their body and their breathing:

"I'm just thinking about the heart and breathing calmly, that was the focus, nothing else"
Several participants reported the focusing and relaxing power of the image, for example:

"My thoughts kept jumping to external thoughts but then I'd sort of get sucked back in and drawn back to the image, and then I just kept starting to relax more and more."

Participants also gave indications that the visuals felt like a mirroring, or extension of their physiological and mental activities, for example at one point a participant is not sure whether they are causing the rings to move or whether the rings are causing them to breathe in a certain way. Interestingly most participants report that they notice changes in the HRV spectrum rays depending on their mental focus, even when they don't fully understand what information the rays are displaying, a point that is discussed further in the next section.

It is clear that the relationship between the visuals and the participants' mental and physical states leads them to a feeling of engagement. This supports previous research by Edmonds et al [5] which proposes a set of requirements for successful engagement in physiologically driven computing and art. These include 1) ease of 
connection to input and 2) immersion in output, which together lead to 3) sense of personal connection with technologies or 'somatic extension'. In the case of Cardiomorphologies there is evidence that this state if 'somatic extension' was achieved, for example:

"I'm feeling [...] I don't know if it's the right word but integrated with the visual, because it's kind of synchronised with how you're thinking and breathing and moving and your heartbeat and everything all integrated together"

\subsection{Reflection}

The majority of participants' experience accorded with the affective goal of Explorative and Curious. The opening sections of their report tend to be characterised by words such as "curious", "fascinated" and "intrigued". They explored the work in three ways; by experimenting with their breathing, with their thoughts or with trying to create certain patterns and shapes with the visuals. For most this process is described in positive terms, but there was one interesting exception. One participant describes a feeling of frustration at how little he could make the visuals do;

"I thought "oh, well I'll try different things and see what I can make this thing do...I looked at the guy before me and I thought "oh he's not doing much maybe I can make it do all sorts of things" but I couldn't"

This coincides with a point made by Edmonds et al that physiological artworks challenge our commonly held notion of control [5]. Edmonds suggests that it is more useful to think of the participant as "influencing" rather than "controlling" physiological systems, in which you are "being" rather than "doing" something in order to have an effect. It is an interesting challenge for the curatorial and interpretative aspects of such artworks to consider how audiences might be encouraged to adopt this different approach to interaction, so as to avoid frustration.

Most participants seemed comfortable to adopt this alternative kind of interaction, and it facilitated a range of reflections about their body and life through both negative and positive images and a recognition of their physiological effect. There were two particularly interesting aspects to this, first that the participants all had only a very vague understanding of the kind of information being displayed by the spectral rays, and second that, nevertheless, the appearance of the rays prompted in most cases the recounting of very intimate and personally meaningful stories as a way of reflecting on the self.

Stories told by participants included thoughts of loved ones and of work, memories of holidays and reflections on health and previous or current bodily states. There was evidence of a strongly perceived correlation between these thoughts (and their associated physical sensations) and the visualisations, for example one participant says:

"I tried to think of my girlfriend, who I haven't seen for three weeks. And I remember the lights were really going out when I was thinking of her"

Another comments:

"[I] kept trying to think of myself as feeling
really good about my body, [imagining myself]
on the bike, and just cycling along, and I
noticed that I could see the effect of that sort of
calmness, um, that came from being on my
bike..."

This evidence supports Gaver et al's suggestions that ambiguity creates space for people to create meaning [18]. They are engaged in the visuals, but not fully immersed - there is a slight distance, created by ambiguity, which encourages active reflection, and requires that, to interpret the interaction, the participants build connections between their own physical and mental experiences and the visualisations they are being shown.

\section{Conclusion}

The aim of the action research project was to realise as far as possible the experiential and affective qualities desired by the artist. The results show that the final visualisations were very effective in this respect. Engagement was achieved not only by creating a close relationship between physical sensation and visual feedback, but also by adding richness that encouraged creative exploration. Ambiguity played an important role in creating room for personal meaning making which encouraged a reflective experience. An important aspect of the affective impact of the visualisations is the attitude of the participant and a challenge for future work with physiologically interactive art is to encourage a mind-set where participants aren't trying to achieve a goal, but rather exploring and learning as they go. 


\section{Acknowledgements}

Thanks to Matthew Connell and Diana Lorentz at the Powerhouse Museum for facilitating the project. This research was partly conducted within the Australasian CRC for Interaction Design, which is established and supported under the Australian Government's Cooperative Research Centres Programme.

\section{References}

[1] C. Paul, Digital art. London: Thames \& Hudson, 2003.

[2] S.Wilson, Information arts : intersections of art, science, and technology.

Cambridge,Mass. London: MIT, 2003.

[3] R. Picard, Affective Computing. Cambridge, Mass: MIT Press, 1997.

[4] T. Bentley, L. Johnston, and K. von Baggo "Affect: Physiological Responses During Computer Use.," presented at OzCHI2003: New directions in interaction, information environments, media and technology, Brisbane, Australia, 2003.

[5] E. Edmonds, D. Everitt, M. Macaulay, and G. Turner, "On physiological computing with an application in interactive art," Interacting with computers, vol. 16, pp. 897-915, 2004.

[6] K. Hook, P. Sengers, G. Andersson "Sense and Sensibility: Evaluation and Interactive Art," presented at Conference on Human factors in computing systems, Ft. Lauderdale, Florida, USA, 2003. [7] K. Richards, "Let the Body Navigate: An interview with George Khut," in RealTime, vol. 66, 2005.

[8] D. Rosenboom, "Biofeedback and the Arts: Results of Early Experiments." Vanouver, BC: Aesthetic Research Cetre of Canada (A.R.C.), 1976.

[9] B. Costello, L. Muller, S. Amitani and E. Edmonds "Understanding the Situated Experience of Interactive Art: Iamascope in beta_space," presented at Interactive Entertainment, Sydney, Australia, 2005. [10] L. Muller and E. Edmonds, "Living Laboratories: Making and Curating Interactive Art," presented at Siggraph, Boston, 2006.
[11] E. Edmonds and L. Muller, "On Creative Engagement," Visual Communications, vol. 5, 2006.

[12] L. A. Suchman and R. H. Trigg, "Understanding Practice: Video as a Medium for Reflection and Design," in Design at Work: Cooperative Design for Computer Systems, J. Greenbaum,M. Kyng Eds. New Jersey: Lawrence Erlbaum Associates, 1991, pp. 65-89.

[13] S.Amitani, K.Hori "Supporting Musical Composition by Externalising the Composer's Mental Space" Journal of Information Processing Society of Japan, vol. 42, pp23692378.

[14] M. Omodei, A. J. Wearing, and McLennan, "Video Cued Recall: A Minimally Reactive Methodology for Understanding, Detecting and Preventing Error in Control of Complex Systems," presented at 21st European Annual Conference of Human Decision Making and Control, Glasgow, 2002.

[15] F. Kensing, K. Halskov Madsen, "Generating Visions, FutureWorkshops and Metaphorical Design," in Design at work : cooperative design of computer systems, J.M. GreenbaumandM. Kyng, Eds. Hillsdale, N.J: L. Erlbaum Associates, 1991, pp. 155-168.

[16] R. McCraty and D. Childre, "Psychophysiological Correlates of Spiritual Experience," Biofeedback, Winter, pp. 13-17, 2001.

[17] H.-S. Song and P. M. Lehrer, "Applied Psychophysiology and Biofeedback," Applied Psychophysiology and Biofeedback, Vol. 28, pp. 14, 2003.

[18] W. Gaver, J. Beaver, and S. Benford, "Ambiguity as a Resource for Design," presented at ACM Conference on Human Factors in Computing Systems, Ft. Lauderdale, Florida, USA, 2003. 